\title{
The Cauchy problem for a second-order nonlinear hyperbolic equation with initial data on a line of parabolicity
}

\author{
John M.S. Rassias
}

In this paper we study the Cauchy problem for the second order nonlinear hyperbolic partial differential equation

(*) $\quad L u=k(y) \cdot H^{2}\left(x, y, u, u_{x}, u_{y}\right) \cdot u_{x x}-u_{y y}=f\left(x, y, u, u_{x}, u_{y}\right)$, with initial conditions

$$
u(x, 0)=r(x), u_{y}(x, 0)=v(x),
$$

where

$$
\begin{aligned}
x \in I & =[a, b], \\
k(y) & =y^{\alpha}(\alpha>0), \\
H & =H\left(x, y, u, u_{x}, u_{y}\right) \in C^{2}(\cdot), \\
f & =f\left(x, y, u, u_{x}, u_{y}\right) \in C^{2}(\cdot),
\end{aligned}
$$

and $|u|,\left|u_{x}\right|,\left|u_{y}\right|<\infty, y \geq 0, r=r(x) \in c^{4}(\cdot)$, $v=v(x) \in C^{4}(\cdot)$.

These conditions on $k, H, f, r$, and $\nu$ are assumed to be satisfied in some sufficiently small neighborhood of the segment $I, y=0$, in the upper half-plane $y>0$.

Received 14 March 1979. 
This paper generalizes the results obtained by N.A. Lar'kin (Differencial'nye Uravnenija 8 (1972), 76-84), who has treated the special case $H=H(x, y, u)$; that is, the quasi-linear hyperbolic equation (*).

\section{Introduction}

In this paper we study the Cauchy problem for the second order nonlinear hyperbolic partial differential equation

$$
L u \equiv K(y) \cdot H^{2}\left(x, y, u, u_{x}, u_{y}\right) \cdot u_{x x}-u_{y y}=f\left(x, y, u, u_{x}, u_{y}\right),
$$

with initial conditions

$$
u(x, 0)=r(x), u_{y}(x, 0)=v(x), x \in I=[a, b],
$$

where $K^{\prime}=K(y)=y^{\alpha}(\alpha>0), H=H\left(x, y, u, u_{x}, u_{y}\right) \neq 0$, $f=f\left(x, y, u, u_{x}, u_{y}\right)$ are all twice continuously differentiable functions defined for $x \in I$ (an interval), $y \geq 0,|u|,\left|u_{x}\right|,\left|u_{y}\right|<\infty$, and $r=r(x), \quad v=v(x)$ are given functions having continuous derivatives up to the fourth order inclusive. These conditions on $K, H, f, r$, and $v$ are assumed to be satisfied in some sufficiently small neighborhood of the segment $I, y=0$, in the upper half-plane $y>0$.

Frankl [8] solved the Cauchy problem for the equation

$$
\begin{gathered}
y \cdot u_{x x}-u_{y y}+a \cdot u_{x}+b \cdot u_{y y}+c \cdot u=0, \\
a=a(x, y), \quad b=b(x, y), c=c(x, y),
\end{gathered}
$$

under the assumption that the coefficients are analytic.

Berezin [1] treated the same problem for the equation

$$
h(x, y) \cdot y^{\alpha} \cdot u_{x x}-u_{y y}+a \cdot u_{x}+b \cdot u_{y}+c \cdot u+f=0,
$$

with restrictions on the coefficients similar to those for $L u=f$, but with the condition $\alpha \in(0,2)$. Starting from a different point of view Bers [2] solved the Cauchy problem for the equation $K(y) \cdot u_{x x}-u_{y y}=0$, where $K=K(y)$ is a continuous monotone increasing function of $y$ with 
$K(0)=0$. A solution to the same problem has been obtained for the equation $K(y) \cdot u_{x x}-u_{y y}=0$ by Germain and Bader [9]. They make the additional assumption that $K(y) \sim c \cdot y$ as $y \rightarrow 0$ and thus make use of Riemann's method. The result of Bers shows that if the lower order terms are absent in an equation such as

$$
h(x, y) \cdot y^{\alpha} \cdot u_{x x}-u_{y y}+a \cdot u_{x}+b \cdot u_{y}+c \cdot u+f=0,
$$

there is no restriction on the rate of growth of the coefficient of $u_{x x}$. On the other hand, Berezin gives an example to show that for $\alpha>2$ the Cauchy problem is not correctly set for the equation

$$
h(x, y) \cdot y^{\alpha} \cdot u_{x x}-u_{y y}+a \cdot u_{x}+b \cdot u_{y}+c \cdot u+f=0 .
$$

Conti [6] has shown that the Cauchy problem for the equation

$$
h(x, y) \cdot y^{\alpha} \cdot u_{x x}-u_{y y}=f\left(x, y, u, u_{x}, u_{y}\right)
$$

is correctly set for the range $\alpha \in(0,2)$, if

$$
y \cdot f_{u_{x}}\left(x, y, u, u_{x}, u_{y}\right) / \sqrt{K} \rightarrow 0
$$

as $y \rightarrow 0$.

Protter [18] showed that the Cauchy problem for the equation $K(y) \cdot h(x, y) \cdot u_{x x}-u_{y y}+a(x, y) \cdot u_{x}+b(x, y) \cdot u_{y}+c(c, y) \cdot u+f(x, y)=0$ is correctly set, if $y \cdot a(x, y) / \sqrt{K} \rightarrow 0$ as $y \rightarrow 0$.

Lick [11], [12], [13] showed that the Cauchy problem for the equation

$$
y^{2 \alpha} \cdot r^{2}(x, y) \cdot u^{2 \beta} \cdot u_{x}^{2 \gamma} \cdot u_{x x}-u_{y y}+a(x, y) \cdot u_{x}+b(x, y) \cdot u_{y}+c(x, y) \cdot u=0 \text {, }
$$

with homogeneous initial conditions, has only the trivial solution.

Besides, he showed that the Cauchy problem for

$$
u_{x}^{2 \gamma} \cdot u_{x x}-u_{y y}+f\left(x, y, u, u_{x}, u_{y}\right)=0 \quad(y>0) \text {, }
$$

with initial conditions $u(x, 0)=0, u_{y}(x, 0)=\phi(x): x \in I=[a, b]$, is correctly set whenever $f_{p}(x, y, u, p, q)=o\left(y^{\gamma-1}\right)$ as $y \rightarrow 0$, 
$p=u_{x}, q=u_{y}$.

Ogawa [15], [16], [17] showed that the Cauchy problem for the equation

$$
r^{2}(x, y) \cdot u^{2 \beta} \cdot u_{x x}-u_{y y}+f=0, f=f\left(x, y, u, u_{x}, u_{y}\right) \quad(y>0),
$$

with the initial conditions $u(x, 0)=0$,

$$
u_{y}(x, 0)=\dot{\phi}(x): x \in I=[a, b]
$$

is well-posed if $f_{p}(x, y, u, p, q)=o\left(y^{\beta-1}\right)$ as $y \rightarrow 0$.

It has also been proved recently by Singer [20] that the (singular) Cauchy problem for the second order, quasi-linear, hyperbolic partial differential equation

$$
y^{2 \alpha} \cdot r^{2}(x, y) \cdot u^{2 \beta} \cdot u_{x}^{2 \gamma} \cdot u_{x x}-u_{y y}+f\left(x, y, u, u_{x}, u_{y}\right)=0 \quad(y>0)
$$

with initial conditions $u(x, 0)=0, u_{y}(x, 0)=\phi(x): x \in I$, has one and only one solution in a neighborhood $(y>0)$ of $I=[a, b]$, if $\alpha, \beta$, and $\gamma$ are non-negative real numbers with $\alpha+\beta+\gamma>0, I$ is any finite interval on the $x$-axis, and $f_{p}(x, y, u, p, q)=o\left(y^{\alpha+\beta+\gamma-1}\right)$ as $y \rightarrow 0, \beta \gamma<1$.

Lar'kin [10] showed that the Cauchy problem for the second-order quasi-linear hyperbolic equation with initial data on a line of parabolicity, namely, for the equation

$$
u_{y y}-y^{m} \cdot k^{2}(x, y, u) \cdot u_{x x}+\Phi(x, y, u)=0, y>0, m>0,
$$

where $K(x, y, u) \neq 0$ and $\Phi(x, y, u)$ are twice continuously differentiable for $x \in[a, b], y \geq 0,|u|<\infty$, with initial data $u(x, 0)=\phi(x), u_{y}(x, 0)=\psi(x): x \in[a, b]=I, y=0, \phi$ and $\psi$ continuously differentiable up to the fourth order inclusive, has a unique regular solution.

In the developments mentioned above the authors have applied mainly Schauder's fixed point theorem to a system of integral equations, the Picard method of iteration, and the Ascoli-Arrela theorem, as well as the 
classical mean value theorem.

\section{THEOREM}

Let us consider the non-linear hyperbolic equation of second order

(I) $L u \equiv K(y) \cdot H^{2}\left(x, y, u, u_{x}, u_{y}\right) \cdot u_{x x}-u_{y y}=f\left(x, y, u, u_{x}, u_{y}\right)$, with initial data on a line of parabolicity; nomely,

$$
\left\{\begin{array}{l}
u(x, 0)=r(x), \\
u_{y}(r, 0)=v(x),
\end{array}\right.
$$

where $H=H\left(x, y, u, u_{x}, u_{y}\right) \neq 0, K(y)=y^{\alpha}(\alpha>0)$, $f=f\left(x, y, u, u_{x}, u_{y}\right)$ are twice continuously differentiable functions defined for $x \in[a, b], y \geq 0,|u|,\left|u_{x}\right|,\left|u_{y}\right|<\infty, i^{2}=r(x)$, $v=v(x)$ are given functions having continuous derivatives up to the fourth order inclusive.

Equation (1) is hyperbolic for $y>0$ and is parabolically degenerate for $y=0$.

If $H=H\left(x, y, u, u_{x}, u_{y}\right), f=f\left(x, y, u, u_{x}, u_{y}\right), r=r(x)$, and $v=v(x)$ satisfy the above conditions in some sufficiently smazl neighborhood of the segment $a \leq x \leq b, y=0$ in the half-plane $y>0$, then the Cauchy problem (1) and (2) has a unique regular solution $u=u(x, y)$ in some sufficiently small neighborhood of the segment $a \leq x \leq b, y=0$ in the half-plane $y>0$, which is twice continuously aifforentiable for $y>0$ and continuous for $y \geq 0$.

\section{Proof}

- Ct us introduce tile ne:s unnnown function

$$
v=u-y \cdot v-r \text {, }
$$

for which the ini,inl conjitions (2) become homogeneous, and for which obviously there is $\mathrm{m}$ loss of generality.

By (3), corditions (1) and (2) become 
(1)' $\bar{L} v \equiv K(y) \cdot H^{2}\left(x, y, v, v_{x}, v_{y}\right) \cdot v_{x x}-v_{y y}=F\left(x, y, v, v_{x}, v_{y}\right)$,

(2)'

$$
\left\{\begin{array}{l}
v(x, 0)=0, \\
v_{y}(x, 0)=0,
\end{array}\right.
$$

where $H=H\left(x, y, v, v_{x}, v_{y}\right)$, and $F=F\left(x, y, v, v_{x}, v_{y}\right)$ are known functions. For convenience, let $u=v, H=K=K\left(x, y, u, u_{x}, u_{y}\right)$ in (1)' and (2)', such that

$$
\overline{\bar{L}}{ }_{u} \equiv y^{\alpha} \cdot K^{2} \cdot u_{x x}-u_{y y}=F\left(x, y, u, u_{x}, u_{y}\right),
$$

(2)"

$$
u(x, 0)=u_{y}(x, 0)=0 \text {. }
$$

(I). At first, we reduce the non-linear hyperbolic partial differential equation of second order, (I)", to a system of integral equations, as follows.

Let us introduce the new unknown functions

(4)

$$
\left\{\begin{array}{l}
u_{1}=u_{1}(x, y)=z_{1}(x, y), \\
u=z_{1}, \\
u_{2}=u_{2}(x, y)=H\left(x, y, u, u_{x}, u_{y}\right) \cdot y^{\alpha / 2} \cdot z_{2}+z_{3}, \\
u_{x}=z_{2}, u_{y}=z_{3} \\
u_{3}=u_{3}(x, y)=-H\left(x, y, u, u_{x}, u_{y}\right) \cdot y^{\alpha / 2} \cdot z_{2}+z_{3}, \\
H=K=K\left(x, y, u, u_{x}, u_{y}\right) .
\end{array}\right.
$$

It is clear that

$$
\left(z_{1}\right)_{y}=z_{3}, \quad\left(z_{2}\right)_{y}=\left(z_{3}\right)_{x},
$$

$$
\begin{gathered}
\left(z_{3}\right)_{y}=y^{\alpha} \cdot k^{2}\left(x, y, u, u_{x}, u_{y}\right) \cdot\left(z_{2}\right)_{x}-F\left(x, y, u, u_{x}, u_{y}\right), \\
z_{1}(x, 0)=z_{2}(x, 0)=z_{3}(x, 0)=0 .
\end{gathered}
$$

By $(4)$, 
The Cauchy problem

351

(4). $\left\{\begin{array}{l}z_{1}=z_{1}(x, y)=u_{1}, \\ z_{2}=z_{2}(x, y)=\left[u_{2}(x, y)-u_{3}(x, y)\right] /\left(2 y \cdot \alpha / 2_{k}\right), \\ z_{3}=z_{3}(x, y)=\left[u_{2}(x, y)+u_{3}(x, y)\right] / 2 ;\end{array}\right.$

(7) $\quad\left(u_{2}\right)_{y}-x \cdot y^{\alpha / 2} \cdot\left(u_{2}\right)_{x}=\left(A_{2} / y\right) \cdot\left(u_{2}-u_{3}\right)+B_{2}$,

where

(8)

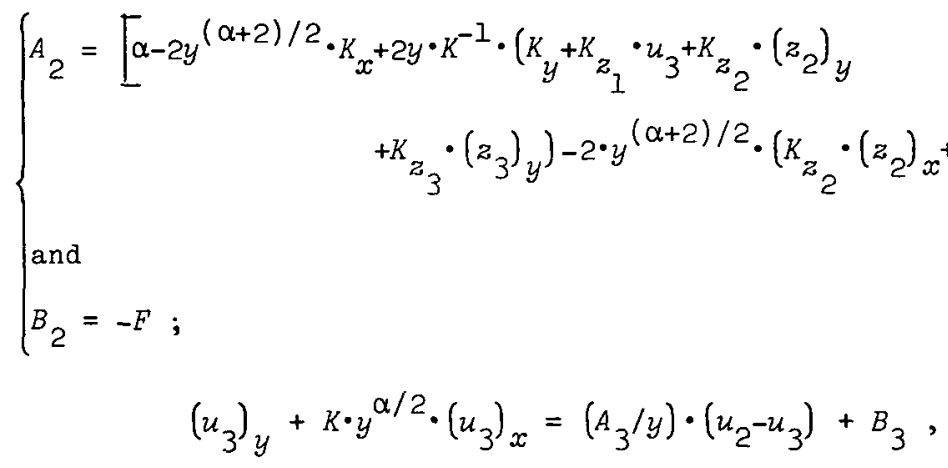

where

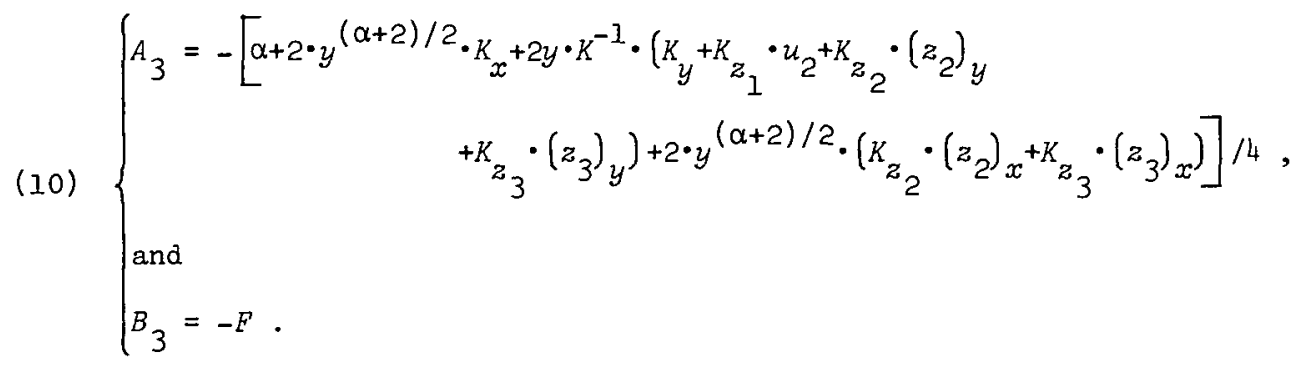

Hence (1)" may be written equivalently as the following system of three equations, namely:

$$
\begin{aligned}
u_{1 y} & =\left[u_{2}(x, y)+u_{3}(x, y)\right] / 2, \\
u_{2 y}-\left(k \cdot y^{\alpha / 2}\right) \cdot u_{2 x} & =\frac{A_{2}}{y} \cdot\left[u_{2}(x, y)-u_{3}(x, y)\right]+B_{2}, \\
u_{3 y}+k \cdot y^{\alpha / 2} \cdot u_{3 x} & =\frac{A_{3}}{y} \cdot\left[u_{2}(x, y)-u_{3}(x, y)\right]+B_{3} ;
\end{aligned}
$$

or

https://doi.org/10.1017/S0004972700011060 Published online by Cambridge University Press 
(11)

$$
\left\{\begin{aligned}
u_{1 y} & =\left(u_{2}+u_{3}\right) / 2, \\
u_{i y}-(-1)^{i} \cdot\left(K \cdot y^{\alpha / 2}\right) \cdot u_{i x} & =\frac{A_{i}}{y}\left(u_{2}-u_{3}\right)+B_{i}(i=1,2) .
\end{aligned}\right.
$$

It is worth noting that

$$
B_{2}=B_{3}=-F=y^{\alpha} \cdot H^{2} \cdot\left(y \cdot v_{x x}+{ }_{x x}\right)-f .
$$

In fact, by (3), $u=v+y \cdot v+r$, whence

$$
\begin{gathered}
u_{x}=v_{x}+y \cdot v_{x}+r_{x}, \quad u_{x x}=v_{x x}+y \cdot v_{x x}+r_{x x}, \\
u_{y}=v_{y}+v, \quad u_{y y}=v_{y y} .
\end{gathered}
$$

Therefore,

$$
\begin{aligned}
K(y) \cdot H^{2} \cdot u_{x x x}-u_{y y} & =K(y) \cdot H^{2} \cdot\left(v_{x x}+y \cdot v_{x x}+r_{x x}\right)-v_{y y} \\
& =\left(K(y) \cdot H^{2} \cdot v_{x x}-v_{y y}\right)+K(y) \cdot H^{2} \cdot\left(y \cdot v_{x x}+r x\right) \\
& =F+K(y) \cdot H^{2} \cdot\left(y \cdot v_{x x}+r_{x x}\right)=f,
\end{aligned}
$$

and thus

$$
E=f\left(x, y, u, u_{x}, u_{y}\right)-y^{\alpha} \cdot H^{2}\left(x, y, u, u_{x}, u_{y}\right) \cdot\left(y \cdot v_{x x}+r_{x x}\right) .
$$

The characteristics of (11) are the lines $x=$ const and the two families of curves given by $d y / d x= \pm y^{-\alpha / 2} \cdot K^{-1}$.

Let $P=P(x, y)$ be a point in $D$ and construct the three characteristics of (11) passing through the point $P$.

The left side of each of the equations in (11) represents a derivative in a characteristic direction.

If we denote by $S_{2}$ the member of the family

$$
\frac{d y}{d x}=-y^{-\alpha / 2} \cdot K^{-1}
$$

passing through $P$, and by $S_{3}$ the member of the family

$$
\frac{d y}{d x}=+y^{-c / 2} \cdot K^{-1}
$$


passing through $P$, we can write (11) in the form

$$
\left\{\begin{array}{l}
\frac{d u_{1}}{d y}=\left(u_{2}+u_{3}\right) / 2, \\
\frac{d u_{i}}{d S_{i}}=\frac{\tilde{A}_{i}}{y} \cdot\left(u_{2}-u_{3}\right)+\tilde{B}_{i} \quad(i=2,3),
\end{array}\right.
$$

where

$$
\tilde{A}_{i}=A_{i} /\left[1+y^{\alpha} \cdot K^{2}\right]^{\frac{2}{2}} \quad(i=1,2), \tilde{B}_{i}=-F /\left[1+y^{\alpha} \cdot k^{2}\right]^{\frac{1}{2}} .
$$

In fact

$$
\frac{d u_{i}}{d S_{i}}=\left(u_{i x} d x+u_{i y} d y\right) / d S_{i}=\left(u_{i y}+u_{i x} \frac{d x}{d y}\right) \cdot \frac{d y}{d S_{i}}=\left[u_{i y}-u_{i x} \cdot k \cdot y^{\alpha / 2}\right] \cdot\left(\frac{d y}{d S_{i}}\right) \text {, }
$$

where

$$
\frac{d y}{d S_{i}}=\left|\frac{d u_{i}}{d S_{i}}\right|=\left[1+y^{\alpha} \cdot k^{2}\right]^{\frac{1}{2}}
$$

By integrating (13) along the characteristics, we obtain the required system of non-linear singular integral equations, equivalent to ( 1 )":

$$
u_{1}(\xi, n)=\frac{1}{2} \cdot \int_{0}^{n}\left[u_{2}(x, y)+u_{3}(x, y)\right] \cdot d y
$$

(14) $u_{i}(\xi, n)=\int_{0}^{n}\left\{\left[\tilde{A}_{i}\left(x_{i}, y, u_{1}, u_{2}, u_{3}\right) / y\right] \cdot\left[u_{2}\left(x_{i}, y\right)-u_{3}\left(x_{i}, y\right)\right]\right.$

$$
\left.+\tilde{B}_{i}\left(x_{i}, y, u_{1}, u_{2}, u_{3}\right)\right\} \cdot d y \quad(i=2,3)
$$

where

$$
x_{i}(y ; \xi, \eta)=\xi-(-1)^{i} \cdot \int_{n}^{y} t^{\alpha / 2} \cdot k\left(x, t, u_{1}, u_{2}, u_{3}\right) \cdot d t
$$$$
(i=2,3) \text {, }
$$

and subject to the initial conditions

$$
u_{i}(x, 0)=0, i=1,2,3, x \in[a, b] .
$$

(II). By applying the above reduction (I), we achieve the main part of the proof of our theorem, as follows. 
Let $\bar{D}=\left\{(x, y) \mid(x \in[a, b])\right.$ and $\left.\left(y \in\left[0, y_{0}\right]\right)\right\}$ be a closed domain in $\mathbb{R}^{2}$, where $y_{0}$ is arbitrarily chosen.

Let us define in $\bar{D}$ the following set of continuous functions, namely:

$C(\bar{D})=\left\{\phi \mid \phi=\phi(x, y)\right.$ is continuous in $\bar{D}$ such that $\phi_{x}$ is equicontinuous with respect to $x$ and $\phi(x, 0)=0$; $\max \left\{|\phi|,\left|\phi_{x}\right|,\left|\phi_{y}\right|\right\} \leq M$, where $M$ is a fixed number $\}$.

Let $\bar{D}_{j}=\bigcap_{i=1}^{3} \bar{D}_{j i}$, where $\bar{D}_{j i}$ are the closed domains of definition of arbitrary functions $\phi_{j i} \in C(\bar{D})$ replacing the functions $u_{i}$ $(i=1,2,3)$ and such that these domains are bounded by

$$
\begin{aligned}
& y=\varepsilon<y_{0}, y=0, \\
& x_{2}=a+\int_{0}^{y} t^{\alpha / 2} \cdot K\left(x, t, \phi_{j 1}(x, t), \phi_{j 2}(x, t), \phi_{j 3}(x, t)\right) \cdot d t,
\end{aligned}
$$

and

$$
x_{3}=b+\int_{0}^{y}(-t)^{\alpha / 2} \cdot K\left(x, t, \phi_{j 1}(x, t), \phi_{j 2}(x, t), \phi_{j 3}(x, t)\right) \cdot d t,
$$

where $i=1,2,3$ and $j=1,2,3, \ldots$, and the function $K=K\left(x, y, \phi_{1}, \phi_{2}, \phi_{3}\right)$ is bounded in the closed parallelepiped $\overline{\mathrm{II}}=\left\{(x, y, \phi) \mid(x \in[a, b])\right.$ and $\left(y \in\left[0, y_{0}\right]\right)$ and

$$
\left.\left.\left(\phi=\phi_{1}, \phi_{2}, \phi_{3}\right) \in[-M, M]\right)\right] \subseteq \mathrm{R}^{3} \text {. }
$$

LEMMA 1. Let $\max _{\bar{D}}|K| \leq \bar{M}$ where $K=K\left(x, y, \phi_{1}, \phi_{2}, \phi_{3}\right)$, $\left|\phi_{i}\right| \leq M \quad(i=1,2,3)$.

Let $\bar{D}_{\varepsilon}=\bigcap_{j=1}^{\infty} D_{j}$, and $S_{\varepsilon}=\left\{\phi \mid \phi=\phi(x, y)\right.$ is defined in $\left.\bar{D}_{\varepsilon}\right\}$, where $\varepsilon>0$ is sufficiently smazl.

We define the norm of continuously differentiable functions 
$\phi=\phi(x, y)$ in $S_{\varepsilon}$ as $\|\phi\|_{c^{I}}=\overline{m a x}_{\varepsilon}\left\{|\phi|,\left|\phi_{x}\right|\right\}$

We prove that the following inequalities hold, namely:

$$
\left|x_{k}^{\phi}(y ; \xi, \eta)-x_{k}^{\psi}(y ; \xi, \eta)\right| \leq \lambda \cdot\|\phi-\psi\| \quad(k=2,3),
$$

where

$$
\begin{aligned}
x_{k}^{\phi}(y ; \xi, \eta) & =\xi-(-1)^{k} \cdot \int_{n}^{y} t^{\alpha / 2} \cdot K\left[x^{\phi}, t, \phi_{1}, \phi_{2}, \phi_{3}\right) \cdot d t, \\
\phi_{i} & =\phi_{i}(x, t) \quad(i=1,2,3),(k=2,3), \\
x_{k}^{\psi}(y ; \xi, \eta) & =\xi-(-1)^{k} \cdot \int_{n}^{y} t^{\alpha / 2} \cdot K\left(x^{\psi}, t, \psi_{1}, \psi_{2}, \psi_{3}\right) \cdot d t, \\
\psi_{i} & =\psi_{i}(x, t) \quad(i=1,2,3),(k=2,3) ;
\end{aligned}
$$

(1.2) $\left|x_{2}(y ; \xi, n)-x_{3}(y ; \xi, n)\right| \leq \mu \cdot n^{(\alpha+2) / 2}, \quad 1 \leq\left|x_{\xi}(y ; \xi, n)\right|<2$,

$$
\left|\frac{\partial x_{2}}{\partial \xi}(y ; \xi, \eta)-\frac{\partial x_{3}}{\partial \xi}(y ; \xi, \eta)\right| \leq \Lambda \cdot \eta(\alpha+2) / 2,
$$

such that

$$
\mu=4 \bar{M} /(\alpha+2), \quad \lambda<1, \quad 0 \leq y \leq \eta \leq \varepsilon,
$$

and

$$
\max _{\bar{D}}\left\{\left|K_{x}\right|,\left|K_{y}\right|,\left|K_{\phi}\right|,\left|K^{-1}\right| ;|\tilde{B}|,\left|\tilde{B}_{x}\right|,\left|\tilde{B}_{\phi}\right|\right\} \leq \bar{M},
$$

where

$$
\tilde{B}=\tilde{B}_{2}=\tilde{B}_{3}, \quad \tilde{B}=\tilde{B}(x, y, \phi), \quad K=K(x, y, \phi) .
$$

Proof. The proof of this lemma is an immediate application of the classical mean-value theorem. We prove only the inequalities (1.1) and (1.2) and observe that (1.3) is clear. In fact,

$$
\begin{aligned}
x_{k}^{\phi}(y ; \xi, n)-x_{k}^{\psi}(y ; \xi, n) & \leq \bar{\mu} \cdot\left|\int_{n}^{y} t^{\alpha / 2} \cdot\left[\left|x^{\phi}-x^{\psi}\right|+|\phi-\psi|\right] \cdot d t\right| \\
& \leq Z \leq \lambda \cdot\|\phi-\psi\|, \lambda<1,
\end{aligned}
$$


because

$$
\tau=\max _{\bar{D}_{\varepsilon}}\left|x_{k}^{\phi}-x_{k}^{\psi}\right|,(\tau+|\phi-\psi|) \cdot|y-n|^{(\alpha+2) / 2} \cdot(2 \bar{M} / \alpha+2) \geq \tau,
$$

and therefore,

$$
2 \cdot\left[1-(2 \bar{M} / \alpha+2) \cdot|y-\eta|^{(\alpha+2) / 2}\right] \leq(2 \bar{M} / \alpha+2) \cdot|y-\eta|^{(\alpha+2) / 2} \cdot\|\phi-\psi\|,
$$

and by letting $\varepsilon$ be so that $0 \leq y \leq n \leq \varepsilon$,

$$
\text { (1.2) } \begin{aligned}
\left|x_{2}(y ; \xi, \eta)-x_{3}(y ; \xi, \eta)\right| & \leq 2 \cdot \int_{0}^{y}\left|t^{\alpha / 2} \cdot K\right| \cdot d t \leq 2 \bar{M} \cdot \int_{0}^{y} t^{\alpha / 2} \cdot d t \\
& \leq 2 \bar{M} \cdot \int_{0}^{\eta} t^{\alpha / 2} \cdot d t \\
& =(4 \bar{M} / \alpha+2) \cdot \eta^{(\alpha+2) / 2}=\mu \cdot \eta{ }^{(\alpha+2) / 2},
\end{aligned}
$$

where $\mu=4 \vec{M} / \alpha+2$.

The rest of the inequalities is clear.

LEMMA 2. For all $n$ the following inequalities hold in $S_{\varepsilon}$, namezy:

$$
\left|u_{i}^{(n)}(\xi, n)\right| \leq \bar{M} \cdot \sum_{j=0}^{n} \delta^{j} \cdot n \quad(i=1,2,3),
$$

$$
\left|u_{2}^{(n)}(\xi, n)-u_{3}^{(n)}(\xi, n)\right| \leq \bar{M} \cdot \sum_{j=0}^{n} \delta^{j} \cdot \eta^{(\alpha+2) / 2},
$$

(2.3) $\left|u_{i}^{(n)}\left(x_{2}, y\right)-u_{i}^{(n)}\left(x_{3}, y\right)\right| \leq \bar{M} \cdot \sum_{j=0}^{n} \delta^{j} \cdot \eta^{(a+2) / 2}(i=1,2,3)$,

where $\delta$ is taken sufficiently close to 1 .

Proof. To establish the existence of a solution of the system (14) we proceed by iterations. We define $u_{i}^{(0)}(\xi, n)=0 \quad(i=1,2,3)$, and the quantities $u_{i}^{(n)}(x, y)$ by the relations 
(i7) $\left\{\begin{array}{r}u_{1}^{(n)}=\frac{1}{2} \cdot \int_{0}^{n}\left[u_{2}^{(n-1)}+u_{3}^{(n-1)}\right] \cdot d y, \\ u_{i}^{(n)}=\int_{0}^{n}\left\{\left|\tilde{A}_{i}\left(x_{i}, y, \phi_{1}, \phi_{2}, \phi_{3}\right) / y\right| \cdot\left[u_{2}^{(n-1)}\left(x_{i}, y\right)-u_{3}^{(n-1)}\left(x_{i}, y\right)\right]\right. \\ \left.+\tilde{B}_{i}\left(x_{i}, y, \phi_{1}, \phi_{2}, \phi_{3}\right)\right\} \cdot d y \quad(i=2,3) .\end{array}\right.$

We proceed by induction on $n$; that is, we show that all the inequalities (2.1), (2.2), and (2.3) hold for $n=1$, and then by assuming they all hold for $n=k$, we establish each inequality for $n=k+1$.

We establish all inequalities simultaneously.

Case $1 . n=1$

(2.1) $\left|u_{i}^{(I)}(\xi, \eta)\right| \leq \int_{0}^{n}\left|\tilde{B}_{i}\left(x_{i}, y, \phi_{1}, \phi_{2}, \phi_{3}\right)\right| \cdot d y \leq \bar{M} \cdot \eta \leq \bar{M} \cdot \sum_{j=0}^{1} \delta^{j} \cdot \eta$ $(i=1,2)$,

where $\delta$ is taken sufficiently close to 1 . The case $i=1$ is trivially true.

$$
\begin{aligned}
\left|u_{2}^{(1)}(\xi, \eta)-u_{3}^{(1)}(\xi, \eta)\right| \leq \int_{0}^{\eta}\left|\tilde{B}_{2}\left(x_{2}, y, \phi\right)-\tilde{B}_{3}\left(x_{3}, y, \phi\right)\right| \cdot d y \\
\quad=\int_{0}^{\eta}\left|\tilde{B}\left(x_{2}, y, \phi\right)-\tilde{B}\left(x_{3}, y, \phi\right)\right| \cdot d y \\
\quad \leq \int_{0}^{\eta}\left[\left\|\tilde{B}_{x}\right\|+\left\|\tilde{B}_{\phi}\right\| \cdot\left\|\phi_{x}\right\|\right] \cdot\left|x_{2}(y ; \xi, \eta)-x_{3}(y ; \xi, \eta)\right| \cdot d y \\
\leq 2 \bar{M} \cdot \mu \cdot M \cdot \eta(\alpha+2) / 2 \cdot \eta \leq \bar{M} \cdot \sum_{j=0}^{1} \delta^{j} \cdot \eta(\alpha+2) / 2,
\end{aligned}
$$

where $\tilde{B}=\tilde{B}_{i} \quad(i=2,3), \delta<1, \varepsilon$ is such that $0 \leq n \leq \varepsilon \quad$ (in $S_{\varepsilon}$ ), and $2 \mu \cdot M \cdot n<1$. Besides, we have applied Lemma 1 through this proof. Similarly 
(2.3)

$$
\begin{aligned}
\left|u_{i}^{(1)}\left(x_{2}, y\right)-u_{i}^{(1)}\left(x_{3}, y\right)\right| & \leq \int_{0}^{\eta} \mid \tilde{B}\left(x_{i}\left(t ; x_{2}, y\right), t, \phi\right) \\
& -\tilde{B}\left(x_{i}\left(t ; x_{3}, y\right), t, \phi\right) \mid \cdot d t \\
\leq & \bar{M} \cdot \sum_{j=0}^{1} \delta^{j} \cdot \eta^{(\alpha+2) / 2} .
\end{aligned}
$$

The case $i=1$ is trivially true.

Case 2. $n=k+1$

We suppose that Lemma 2 holds for $n=k$.

(2.1) $\left|u_{1}^{(k+1)}(\xi, n)\right| \leq \frac{2}{2} \int_{0}^{n}\left[\left|u_{2}\right|^{k}+\left|u_{3}\right|^{k}\right] \cdot d y \leq \frac{2}{2} \int_{0}^{n}\left[2 \bar{M} \cdot \sum_{j=0}^{k} \delta^{j} y\right] d y$

$$
=\bar{M} \cdot \sum_{j=0}^{k} \delta^{j} \cdot \frac{3}{2} n^{2} \leq \bar{M} \cdot \sum_{j=0}^{k+1} \delta^{j} \cdot n,
$$

$$
\begin{aligned}
\left|u_{i}^{(k+1)}(\xi, n)\right| \leq \int_{0}^{n}\left\{\left[\left|\tilde{A}_{i}\left(x_{i}, y, \phi\right) / y\right|\right] \cdot\left[\left|u_{2}^{(k)}\left(x_{i}, y\right)-u_{3}^{(k)}\left(x_{i} y\right)\right|\right]\right. & \\
& \left.+\left|\tilde{B}_{i}\left(x_{i}, y, \phi\right)\right|\right\} \cdot d y .
\end{aligned}
$$

But

$$
\left|\tilde{A}_{i}\right|=\left|A_{i} \cdot\right| 1+\left.y^{\alpha} \cdot k^{2}\right|^{-\frac{\alpha}{2}} \mid \leq \frac{\alpha}{4}+\gamma \cdot y \rightarrow \frac{\alpha}{4} \text {, as } y \rightarrow 0 \quad(i=2,3) .
$$

Therefore,

$$
\begin{aligned}
\left|u_{i}^{(k+1)}(\xi, n)\right| & \leq \int_{0}^{n}\left[\left(\frac{\alpha}{4}+\gamma \cdot y\right) \cdot \bar{M} \cdot \sum_{j=0}^{k} \delta^{j} \cdot y^{\alpha / 2}+\bar{M}\right] \cdot d y \\
& \leq \bar{M} \cdot\left[1+\left(\sum_{j=0}^{k} \delta^{j}\right) \cdot \delta\right] \cdot n \leq \bar{M} \cdot \sum_{j=0}^{k+1} \delta^{j} \cdot \eta \quad(i=2,3) ;
\end{aligned}
$$

by choosing the width $\varepsilon$ of the strip so that for $0 \leq y \leq n \leq \varepsilon$,

$$
\left(\frac{\alpha}{4}+\gamma \cdot y\right) \cdot y^{\alpha / 2} \leq \delta<1 \text {, and } \gamma \cdot y \rightarrow 0 \text {, as } y \rightarrow 0 .
$$


(2.2) $\left|u_{2}^{(k+1)}(\xi, n)-u_{3}^{(k+1)}(\xi, n)\right|$

$$
\begin{aligned}
\leq & \int_{0}^{n} \mid\left\{\tilde{A}_{2}\left(x_{2}, y, \phi\right) \cdot\left[u_{2}^{(k)}\left(x_{2}, y\right)-u_{3}^{(k)}\left(x_{3}, y\right)\right]\right. \\
& \left.-\tilde{A}_{3}\left(x_{3}, y, \phi\right) \cdot\left[u_{2}^{(k)}\left(x_{3}, y\right)-u_{3}^{(k)}\left(x_{3}, y\right)\right]\right\} / y \\
& +\tilde{B}_{2}\left(x_{2}, y, \phi\right)-\tilde{B}_{3}\left(x_{3}, y, \phi\right) \mid \cdot d y \\
\leq & \int_{0}^{n}\left\{\left(\frac{\alpha}{4}+\gamma \cdot y\right) \cdot \bar{M} \cdot \sum_{j=0}^{k} \delta^{j} \cdot y^{\alpha / 2}+2 \bar{M} \cdot M \cdot\left|x_{2}-x_{3}\right|\right\} \cdot d y \\
\leq & \bar{M} \cdot \eta(\alpha+2) / 2 \cdot\left\{2 \mu \cdot M \cdot \eta+[4 /(\alpha+2)] \cdot\left\{\frac{\alpha}{4}+\gamma \cdot y\right\} \cdot \sum_{j=0}^{k} \delta^{j}\right\} \\
\leq \bar{M} \cdot \sum_{j=0}^{k+1} \delta^{j} \cdot \eta &
\end{aligned}
$$

by choosing $\varepsilon$ such that $0 \leq n \leq \varepsilon$, and

$$
\begin{gathered}
2 \mu \cdot M \cdot n=2 \cdot \frac{4 \bar{M}}{\alpha+2} \cdot M \cdot n=8 \cdot \frac{\bar{M} \cdot M}{\alpha+2} \cdot \eta<1, \\
\frac{4}{\alpha+2} \cdot\left(\frac{\alpha}{4}+\gamma \cdot y\right) \leq \delta<1, \lim _{y \rightarrow 0} \gamma \cdot y=0 .
\end{gathered}
$$

Similarly,

$$
\begin{aligned}
\left|u_{i}^{(k+1)}\left(x_{2}, y\right)-u_{i}^{(k+1)}\left(x_{3}, y\right)\right| & \int_{0}^{n} \mid\left\{[ \tilde { A } _ { i } ( x _ { i } ( t ; x _ { 2 } , y ) , y , \phi ) / y ] \cdot \left[u_{2}^{(i)}\left(x_{i}\left(t ; x_{2}, y\right), y\right)\right.\right. \\
& \left.-u_{3}^{(k)}\left(x_{i}\left(t ; x_{2}, y\right), y\right)+\tilde{B}_{i}\left(x_{i}\left(t ; x_{2}, y\right), y, \phi\right)\right\} \\
& -\left\{[ \tilde { A } _ { i } ( x _ { i } ( t ; x _ { 3 } , y ) , y , \phi ) / y ] \cdot \left[u_{2}^{(k)}\left(x_{i}\left(t ; x_{3}, y\right), y\right)\right.\right. \\
& \left.-u_{3}^{(k)}\left(x_{i}\left(t ; x_{3}, y\right), y\right)+\tilde{B}_{i}\left(x_{i}\left(t ; x_{3}, y\right), y, \phi\right)\right\} \mid \cdot d y \\
\leq \bar{M} \cdot & \sum_{j=0}^{k+1} \delta^{j} \cdot \eta(\alpha+2) / 2,(i=2,3) .
\end{aligned}
$$

The case $i=1$ is trivial. //

LEMMA 3. For all $n$ the following inequalities hold: 
(3.1) $\left|u_{i}^{(n+1)}(\xi, n)-u_{i}^{(n)}(\xi, n)\right| \leq \bar{M} \cdot \delta^{n} \cdot \eta \quad(i=1,2,3)$;

(3.2) $\left|u_{2}^{(n+1)}(\xi, n)-u_{3}^{(n+1)}(\xi, n)-u_{2}^{(n)}(\xi, n)+u_{3}^{(n)}(\xi, n)\right| \leq \bar{M} \cdot \delta^{n} \cdot \eta^{(\alpha+2) / 2}$;

(3.3) $\left|u_{i}^{(n+1)}\left(x_{2}, y\right)-u_{i}^{(n+1)}\left(x_{3}, y\right)-u_{i}^{(n)}\left(x_{2}, y\right)+u_{i}^{(n)}\left(x_{3}, y\right)\right|$

$$
\leq \bar{M} \cdot \delta^{n} \cdot \eta^{(\alpha+2) / 2} \quad(i=1,2,3) \text {. }
$$

Proof. We prove only (3.1) and (3.2), while (3.3) can be proved in the same way as in the cases (3.1) and (3.2). In fact, by induction on $n$,

$$
\begin{aligned}
& \text { (3.1) }\left|u_{i}^{(n+1)}(\xi, n)-u_{i}^{(n)}(\xi, n)\right| \\
& \leq \int_{0}^{n}\left\{| \tilde { A } _ { i } ( x _ { i } , y , \phi ) / y | \cdot \left[\left|u_{2}^{(n)}\left(x_{i}, y\right)-u_{2}^{(n-1)}\left(x_{i}, y\right)\right|\right.\right. \\
& \left.\left.+\left|u_{3}^{(n)}\left(x_{i}, y\right)-u_{3}^{(n-1)}\left(x_{i}, y\right)\right|\right]\right\} \cdot d y \\
& \leq \int_{0}^{n} 2\left(\frac{\alpha}{4}+\gamma \cdot y\right) \cdot \frac{1}{y} \bar{M} \cdot \delta^{n-1} \cdot y \cdot d y \\
& \leq \bar{M} \cdot \delta^{n} \cdot n, \lim _{y \rightarrow 0} \gamma \cdot y=0 \\
& \text { (3.2) }\left|u_{2}^{(n+1)}(\xi, n)-u_{3}^{(n+1)}(\xi, n)-u_{2}^{(n)}(\xi, n)+u_{3}^{(n)}(\xi, n)\right| \\
& \leq \int_{0}^{n} \mid\left[\tilde{A}_{2}\left(x_{2}, y, \phi\right) / y\right] \cdot\left[u_{2}^{(n)}\left(x_{2}, y\right)-u_{3}^{(n)}\left(x_{2}, y\right)\right] \\
& -\left[\tilde{A}_{3}\left(x_{3}, y, \phi\right) / y\right] \cdot\left[u_{2}^{(n)}\left(x_{3}, y\right)-u_{3}^{(n)}\left(x_{3}, y\right)\right] \\
& -\left[\tilde{A}_{2}\left(x_{2}, y, \phi\right) / y\right] \cdot\left[u_{2}^{(n-1)}\left(x_{2}, y\right)-u_{3}^{(n-1)}\left(x_{2}, y\right)\right] \\
& +\left[\tilde{A}_{3}\left(x_{3}, y, \phi\right) / y\right] \cdot\left[u_{2}^{(n-1)}\left(x_{3}, y\right)-u_{3}^{(n-1)}\left(x_{3}, y\right)\right] \mid \cdot d y \\
& \leq \int_{0}^{n}\left(\frac{\alpha}{4}+\gamma \cdot y\right) \cdot \frac{1}{y} \cdot\left\{\mid u_{2}^{(n)}\left(x_{2}, y\right)-u_{3}^{(n)}\left(x_{2}, y\right)\right. \\
& -u_{2}^{(n-1)}\left(x_{2}, y\right)+u_{3}^{(n-1)}\left(x_{2}, y\right)|+| u_{2}^{(n)}\left(x_{3}, y\right) \\
& \left.-u_{3}^{(n)}\left(x_{3}, y\right)-u_{2}^{(n-1)}\left(x_{3}, y\right)+u_{3}^{(n-1)}\left(x_{3}, y\right) \mid\right\} \cdot d y \\
& \leq 2 \cdot\left(\frac{\alpha}{4}+\tilde{\alpha}\right) \cdot \bar{M} \cdot \delta^{n-1} \cdot \eta^{\alpha / 2} \leq \bar{M} \cdot \delta^{n} \cdot n^{(\alpha+2) / 2}
\end{aligned}
$$




$$
\tilde{\alpha}=\tilde{\alpha}(y)=\gamma \cdot y, \lim _{y \rightarrow 0} \tilde{\alpha}(y)=0 .
$$

(3.3) follows similarly, as in the above case. //

Then by applying Lemmas $1,2,3$, and Ascoli-Arzela's theorem we get the required result. (See also [10].)

In fact, by Lemma 3 it is clear that the sequences $\left\{u_{i}^{(n)}(x, y)\right\}$ $(i=2,3)$ converge uniformiy in $S_{\varepsilon}$. Since each $u_{i}^{(n)}=u_{i}^{(n)}(x, y)$ is continuous in $S_{E}$, so are the limits, which we denote by $u_{i}=u_{i}(x, y)$.

The resulting linear system is solvable, and the solutions satisfy the following inequalities uniformly in $S_{\varepsilon}$ :

$$
\left|u_{i}(x, y)\right| \leq \bar{M} \cdot \sum_{j=0}^{\infty} \delta^{j} \cdot y
$$

(*) $\left|u_{2}(x, y)-u_{3}(x, y)\right| \leq M \cdot \sum_{j=0}^{\infty} \delta^{j} \cdot y^{(\alpha+2) / 2}$, where $\delta<1, i=1,2,3$. For an appropriate choice of $\varepsilon,\left|u_{i}(\xi, \eta)\right| \leq M$ for all $\phi \in S_{\varepsilon}$.

Similar inequalities hold for the derivatives $\left(u_{i}\right)_{\xi}(\xi, \eta)$ and $\left(u_{i}\right)_{\eta}(\xi, n)$ in $S_{\varepsilon}(i=1,2,3)$. Moreover, if $\varepsilon$ is sufficiently small, $\left|\left(u_{i}\right)_{\xi}\right| \leq M,\left|\left(u_{i}\right)_{\eta}\right| \leq M$ in $s_{\varepsilon}(i=1,2,3)$.

Then by taking into account (14), (15), and (16), we are done.

As a matter of fact to prove that the system of integral equations (14) has a unique solution in a neighborhood $(y>0)$ of $I=[\alpha, \beta]$, we apply the well-known Schauder Fixed Point Theorem (namely: a continuous mapping of a convex, compact subset of a Banach space into itself has a fixed point).

Let the continuous operator $T: S_{\varepsilon} \stackrel{\text { into }}{\longrightarrow} S_{\varepsilon}$ be defined as follows: 


$$
\left\{\begin{array}{r}
T_{1}(\phi)=\frac{1}{2} \cdot \int_{0}^{n}\left[u_{2}(x, y)+u_{3}(x, y)\right] \cdot d y, \\
T_{i}(\phi)=\int_{0}^{n}\left\{\left[\tilde{A}_{i}\left(x_{i}^{\phi}, x, \phi\right) / y\right] \cdot\left[u_{2}\left(x_{i}^{\phi}, y\right) \cdot \cdot u_{3}\left(x_{i}^{\phi}, y\right)\right]+\tilde{B}_{i}\left(x_{i}^{\phi}, y, \phi\right)\right\} \cdot d y \\
(i=2,3),
\end{array}\right.
$$

where $\phi=\phi\left(x_{i}^{\phi}, y\right)$.

By applying the classical mean value theorem and the above lemmas, we get

$$
\begin{aligned}
& \left\|T_{i}(\phi)-T_{i}(\psi)\right\| \leq \lambda \cdot \eta \cdot\|\phi-\psi\|, \\
& \left\|T_{I}(\phi)-T_{I}(\psi)\right\| \leq \lambda \cdot \frac{\eta^{2}}{2} \cdot\|\phi-\psi\|, \quad i=2,3, \lambda=\text { const. }
\end{aligned}
$$

Therefore

$$
\|T(\phi)-T(\psi)\| \leq \bar{\lambda} \cdot\|\phi-\psi\|,
$$

where $\bar{\lambda}=\lambda \cdot\left(4 \eta+\eta^{2}\right) / 2$; and now we choose $\varepsilon$ sufficiently small, such that $0 \leq y \leq n \leq \varepsilon \Rightarrow \bar{\lambda}<1$; and hence by (19),

$$
T: S_{\varepsilon} \stackrel{\text { into }}{\longrightarrow} S_{\varepsilon} \text { is a contraction operator; }
$$

and from Schauder's Fixed Point Theorem it follows that $T$ has a unique fixed point in $S_{\varepsilon}$.

We note the uniform convergence of $\left\{u_{i}^{(j)}\right\}(i=1,2,3)$, $(j=1,2,3, \ldots)$, and of the derivatives of $\left\{u_{i}^{(j)}\right\}$, namely $\left\{u_{i \xi}^{(j)}\right\}$ and $\left\{u_{i n}^{(j)}\right\}$, in $S_{\varepsilon}$ is a consequence of Ascoli-Arzela's Theorem. For a more detailed proof see also [10]. // 


\section{References}

[1] Н.C. Јерезин [I.S. Berezin], "О задаче Ноши для линейного уравнения второго порядна с начальнымн даннымн на линни параболичности" [On Cauchy's problem for linear equations of the second order with initial conditions on a parabolic line], Mat. Sb. 24 (66) (1949), 301-320.

[2] L. Bers, "On the continuation of a potential gas flow across the sonic line" (NACA Technical Note 2058, 1950).

[3] Garrett Birkhoff, Gian-Carlo Rota, Ordinary differential equations (Ginn, Boston, New York, Chicago, Atlanta, Dallas, Palo Alto, Toronto, 1962).

[4] A.V. Bitsadze, Equations of the mixed type (translated by P. Zador. Macmillan, New York, 1964).

[5] Earl A. Coddington and Norman Levinson, Theory of ordinary differential equations (McGraw-Hill, New York, Toronto, London, 1955).

[6] Roberto Conti, "Sul problema di Cauchy per l'equazione

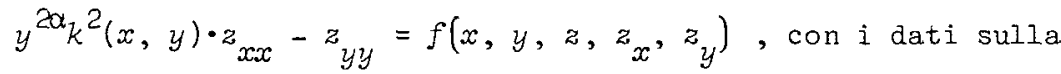
linea parabolica", Ann. Mat. Pura Appl. 31 (1950), 303-326.

[7] Richard Courant and Peter Lax, "On nonlinear partial differential equations with two independent variables", Comm. Pure Appl. Math. 2(1949), 255-273.

[8] Ф.И. Франнль [F.I. Frank|'], "0 задаче Ноши для уравнений смешанного зллнптино-гиперболнчесного типа с начальными данными на пеоеходной линин" [On Cauchy's problem for partial differential equations of mixed elliptic-kyperbolic type with initial data on the parabolic line], Bulz. Acad. Sci. URSS Ser. Math. [Izv. Akad. Nauk SSSR] 8 (1944), 195-224.

[9] P. Germain et R. Bader, "Solutions élémentaires de certaines équations aux dérivées partielles du type mixte", Bulz. Soc. Math. France 81 (1953), 145-174. 
[10] H.A. Ларьнин [N.A. Lar'kin], "O задаче Ношн для нвазилинейного гиперболичесного уравнення второго порядна с начальными данными на линин параболичности" [The Cauchy problem for a second order quasilinear hyperbolic equation with initial data on the curve of parabolicity], Differencial'nye Uravnenija 8 (1972), 76-84.

[11] Dale W. Lick, "A uniqueness theorem for a singular Cauchy problem", Ann. Mat. Pura App2. (4) 72 (1966), 267-274.

[12] Dale W. Lick, "A quasi-linear singular Cauchy problem", Arn. Mat. Pura App 2. (4) 74 (1966), 113-128.

[13] Dale W. Lick, "A singular Cauchy problem", J. Math. AnaZ. Appz. 28 (1969), 93-99.

[14] М. Мередов [M. Meredov], "Об однозначной разрешимостн эадачи Дарбу для одной вырондающейся системы" [The unique solvability of the Darboux problem for a certain degenerate system], Differencial'nye Uraunenija 10 (1974), 89-99.

[15] Hajimu Ogawa, "The singular Cauchy problem for a quasi-linear hyperbolic equation of second order", J. Math. Mech. 12 (1963), $847-856$.

[16] Hajimu Ogawa, "On the unique solution of the singular Cauchy problem for a quasi-linear equation", Ann. Mat. Pura Appl. (4) 66 $(1964), 391-403$.

[17] Hajimu Ogawa, "A nonlinear singular Cauchy problern", J. Math. Anal. App 2. 13 (1966), 527-535.

[18] M.H. Protter, "The Cauchy problem for a hyperbolic second order equation with data on the parabolic line", Conad. J. Math. 6 (1954), 542-553.

[19] J. Schauder, "Der Fixpunktsatz in Funktionalräumen", Studia Math. 2 (1930), 171-180.

[20] Seymour Singer, "The singular Cauchy problem for a nonlinear hyperbolic equation" (PhD thesis, University of California, Berkeley, 1969). 
[21] C.A. Терсенов [S.A. Tersenov], "Н теории типерболичесних уравнений С данньми на линии вырондения типа" [On the theory of hyperbolic equations with data on a line of degeneration type], Sibirsk. Mat. Z. 2 (I961), 913-935.

11 Dervenakion Str,

Daphne,

Athens,

Greece. 